\title{
GABRIELE NEBE
} BORIS VENKOV

\section{The strongly perfect lattices of dimension 10}

Journal de Théorie des Nombres de Bordeaux, tome 12, $\mathrm{n}^{\circ} 2$ (2000), p. 503-518

<http://www.numdam.org/item?id=JTNB_2000_12_2_503_0>

(C) Université Bordeaux 1, 2000, tous droits réservés.

L'accès aux archives de la revue «Journal de Théorie des Nombres de Bordeaux » (http://jtnb.cedram.org/) implique l'accord avec les conditions générales d'utilisation (http://www.numdam.org/conditions). Toute utilisation commerciale ou impression systématique est constitutive d'une infraction pénale. Toute copie ou impression de ce fichier doit contenir la présente mention de copyright.

\section{Numdam}

Article numérisé dans le cadre du programme

Numérisation de documents anciens mathématiques

http://www.numdam.org/ 
Journal de Théorie des Nombres

de Bordeaux 12 (2000), 503-518

\title{
The strongly perfect lattices of dimension 10
}

\author{
par GABRIELE NEBE et BoRIS VENKOV \\ Dedicated to Prof. Jacques Martinet
}

\begin{abstract}
RÉSUMÉ. Cet article donne une classification des réseaux fortement parfaits en dimension 10. A similitude près il y a deux tels réseaux, $K_{10}^{\prime}$ et son réseau dual.

ABSTRACT. This paper classifies the strongly perfect lattices in dimension 10. There are up to similarity two such lattices, $K_{10}^{\prime}$ and its dual lattice.
\end{abstract}

\section{Introduction}

This paper is a continuation of a series of papers on lattices and spherical designs ([Ven], [BaV], [Mar1]). For the basic definitions we refer to these papers and to the books [Mar] and [CoS].

We study lattices, in Euclidean space, whose minimal vectors form a spherical 4-design. Such lattices are called strongly perfect lattices. They form an interesting class of lattices. In particular strongly perfect lattices are perfect lattices in the sense of Voronoi (cf. [Mar]). In small dimensions there are only few such lattices and a complete classification was known up to dimension 9 and for dimension 11. Up to similarity they are root lattices of types $A_{1}, A_{2}, D_{4}, E_{6}, E_{7}, E_{8}$ and their duals ([Ven]). There are no such lattices in dimensions $3,5,9$, and 11 . In this paper we classify the strongly perfect lattices in dimension 10 . This is the first dimension where less trivial lattices do appear. Up to similarity there are exactly two such lattices $K_{10}^{\prime}$ and its dual lattice (cf. Theorem 3.1). This was conjectured in [Ven]. For a description of these lattices we refer to [Mar, Chap.VIII, paragraphe 5].

\section{Some general equations}

2.1. General notation. For a lattice $\Lambda$ in $n$-dimensional Euclidean space we denote by $\Lambda^{*}$ its dual lattice. Important invariants of the lattice $\Lambda$ are its 
determinant $\operatorname{det}(\Lambda)$, its minimum $\min (\Lambda)$ and its Hermite invariant, which is defined as

$$
\gamma(\Lambda):=\frac{\min (\Lambda)}{\operatorname{det}(\Lambda)^{1 / n}} .
$$

The Hermite invariant is an invariant of the similarity class of $\Lambda$. As a function of $\Lambda, \gamma$ is bounded by a constant $\gamma_{n}:=\max \{\gamma(\Lambda) \mid \Lambda \subset$ $\mathbb{R}^{n}$ is a lattice $\}$ that depends only on the dimension $n$. The exact values for $\gamma_{n}$ are known for $n \leq 8$. For higher dimensions, only upper bounds for $\gamma_{n}$ are known. We use Rogers' bound, which gives that $\gamma_{10} \leq 2.2752$ ([CoS, Table 1.2]). Closely related to the Hermite invariant is the Bergé-Martinet invariant

$$
\left(\gamma^{\prime}\right)^{2}(\Lambda):=\gamma(\Lambda) \gamma\left(\Lambda^{*}\right)=\min (\Lambda) \min \left(\Lambda^{*}\right)
$$

which takes into account also the dual lattice.

There are also general bounds on the number of shortest vectors, the Kissing number, of an $n$-dimensional lattice. For $n=10$ the bound is $2 \cdot 297$ (see [CoS, Table 1.5]).

For $a \in \mathbb{R}, a>0$ we let

$$
\Lambda_{a}:=\{x \in \Lambda \mid(x, x)=a\} .
$$

For $\alpha \in \Lambda^{*}, m:=\min (\Lambda)$ and $i \in \mathbb{N}$ let

$$
N_{i}(\alpha):=\left\{x \in \Lambda_{m} \mid(\alpha, x)=i\right\}
$$

and $n_{i}(\alpha):=\left|N_{i}(\alpha)\right|$.

If $\Lambda$ is an integral lattice, that is $\Lambda \subset \Lambda^{*}$, we let for $p \in \mathbb{N}$

$$
\Lambda_{(p)}:=\{v \in \Lambda \mid(v, v) \equiv 0 \quad(\bmod p)\} .
$$

In general $\Lambda_{(p)}$ is only a subset of $\Lambda$, but in many cases that we consider, it turns out to be a sublattice of $\Lambda$.

2.2. Designs and strongly perfect lattices. Let $\left(\mathbb{R}^{n},(),\right)$ be the Euclidean space of dimension $n$. In this section we assume that $m \in \mathbb{R}, m>0$ and $X \subseteq S^{n-1}(m)=\left\{y \in \mathbb{R}^{n} \mid(y, y)=m\right\}$ is such that $X \cap-X=\emptyset$ and $X \cup-X$ is a spherical 4-design. Let $s:=|X|$.

Then by the definition of designs in [Ven] one has for all $\alpha \in \mathbb{R}^{n}$ :

$$
\sum_{x \in X}(x, \alpha)^{2}=\frac{s m}{n}(\alpha, \alpha)
$$

and

$$
\sum_{x \in X}(x, \alpha)^{4}=\frac{3 s m^{2}}{n(n+2)}(\alpha, \alpha)^{2}
$$


Substituting $\alpha:=\xi_{1} \alpha_{1}+\xi_{2} \alpha_{2}$ in (D2) and comparing coefficients, one finds

$$
\text { (D11) } \sum_{x \in X}\left(x, \alpha_{1}\right)\left(x, \alpha_{2}\right)=\frac{s m}{n}\left(\alpha_{1}, \alpha_{2}\right) \text { for all } \alpha_{1}, \alpha_{2} \in \mathbb{R}^{n} \text {. }
$$

Writing $\alpha$ as a linear combination of 4 vectors, (D4) implies that for all $\alpha_{1}, \ldots, \alpha_{4} \in \mathbb{R}^{n}$

$$
\sum_{x \in X}\left(x, \alpha_{1}\right)\left(x, \alpha_{2}\right)\left(x, \alpha_{3}\right)\left(x, \alpha_{4}\right)=
$$

$$
=\frac{s m^{2}}{n(n+2)}\left(\left(\alpha_{1}, \alpha_{2}\right)\left(\alpha_{3}, \alpha_{4}\right)+\left(\alpha_{1}, \alpha_{3}\right)\left(\alpha_{2}, \alpha_{4}\right)+\left(\alpha_{1}, \alpha_{4}\right)\left(\alpha_{2}, \alpha_{3}\right)\right)
$$

In particular

$$
\sum_{x \in X}\left(x, \alpha_{1}\right)\left(x, \alpha_{2}\right)^{3}=\frac{3 s m^{2}}{n(n+2)}\left(\alpha_{1}, \alpha_{2}\right)\left(\alpha_{2}, \alpha_{2}\right)
$$

$$
\sum_{x \in X}\left(x, \alpha_{1}\right)^{2}\left(x, \alpha_{2}\right)^{2}=\frac{s m^{2}}{n(n+2)}\left(2\left(\alpha_{1}, \alpha_{2}\right)^{2}+\left(\alpha_{1}, \alpha_{1}\right)\left(\alpha_{2}, \alpha_{2}\right)\right)
$$

Lemma 2.1. Let $\alpha \in \mathbb{R}^{n}$ be such that $(x, \alpha) \in\{0, \pm 1, \pm 2\}$ for all $x \in X$. Let $N_{2}(\alpha):=\{x \in X \cup-X \mid(x, \alpha)=2\}$ and put

$$
c:=\frac{s m}{6 n}\left(\frac{3 m}{n+2}(\alpha, \alpha)-1\right) \text {. }
$$

Then $\left|N_{2}(\alpha)\right|=c(\alpha, \alpha) / 2$ and

$$
\sum_{x \in N_{2}(\alpha)} x=c \alpha
$$

Proof. Put $\alpha_{2}=\alpha$ in $(D 11)$ and $(D 13)$. Then $(D 13)-(D 11)$ reads as

$$
\sum_{x \in X}(x, \gamma)(x, \alpha)\left((x, \alpha)^{2}-1\right)=(\alpha, \gamma) \frac{s m}{n}\left(\frac{3 m}{n+2}(\alpha, \alpha)-1\right) \text { for all } \gamma \in \mathbb{R}^{n} \text {. }
$$

The left hand side is

and hence

$$
6 \sum_{x \in N_{2}(\alpha)}(x, \gamma)
$$

$$
\left(\sum_{x \in N_{2}(\alpha)} x-c \alpha, \gamma\right)=0
$$

for all $\gamma \in \mathbb{R}^{n}$, where $c$ is the constant of the lemma. Taking the scalar product with $\alpha$, one sees that $2\left|N_{2}(\alpha)\right|=c(\alpha, \alpha)$.

Recall that a lattice $L \subset \mathbb{R}^{n}$ is called strongly perfect, if its minimal vectors form a 4-design. 
Lemma 2.2 ([Ven, Théorème 10.4]). Let $L$ be a strongly perfect lattice of dimension n. Then the Bergé-Martinet invariant

$$
\left(\gamma^{\prime}\right)^{2}(L)=\min (L) \min \left(L^{*}\right) \geq \frac{n+2}{3} .
$$

Proof. Let $m:=\min (L), 2 s:=\left|L_{m}\right|, m^{\prime}:=\min \left(L^{*}\right)$ and and $r:=m m^{\prime}$. For $\alpha \in\left(L^{*}\right)_{m^{\prime}}$ let $n_{i}:=\left|\left\{x \in L_{m} \mid(x, \alpha)= \pm i\right\}\right|$. Then by (D2) and (D4) $\sum_{i=1}^{r} i^{2} n_{i}=\frac{s r}{n}$ and $\sum_{i=1}^{r} i^{4} n_{i}=\frac{3 s r^{2}}{n(n+2)}$. The difference is

$$
\sum_{i=2}^{r}\left(i^{4}-i^{2}\right) n_{i}=\frac{r s}{n}\left(\frac{3 r}{n+2}-1\right) .
$$

Since the left hand side is a sum of non negative numbers, the right hand side is non negative and therefore $r \geq \frac{n+2}{3}$. One also sees that equality implies that $n_{2}=n_{3}=\ldots=0$.

2.3. Gauss sums and the Milgram-Braun formula. For the classification of (strongly perfect) lattices it is helpful to know restrictions on the possible genera of even lattices. Let $L$ be an even lattice in the Euclidean space $\left(\mathbb{R}^{n},(),\right)$ and $L^{*}$ be its dual lattice. Then the bilinear form $($,$) induces$ a quadratic form $q: L^{*} / L \rightarrow \mathbb{R} / \mathbb{Z}$ on the finite abelian group $D:=L^{*} / L$ by $q(x+L):=\frac{1}{2}(x, x)+\mathbb{Z}$. Then the Gauss sum $G(L)=G(D, q)$ is defined as

$$
G(D, q):=\frac{1}{\sqrt{|D|}} \sum_{d \in D} \exp (2 \pi i q(d)) .
$$

The following is known as the Milgram-Braun formula.

Lemma 2.3 (see [Scha, Corollary 5.8.2], [MiH, Appendix 4]).

$$
G(L)=\exp \left(\frac{2 \pi i}{8}\right)^{n} .
$$

Moreover if $(D, q)=\left(D_{1}, q_{1}\right) \perp\left(D_{2}, q_{2}\right)$ then $G(D, q)=G\left(D_{1}, q_{1}\right)$. $G\left(D_{2}, q_{2}\right)$. Since $D$ is the orthogonal sum of its Sylow $p$-subgroups, and $G(L)$ is independent of the even lattice $L$, it suffices to calculate $G(D, q)$ for anisotropic orthogonally indecomposable $p$-groups.

Lemma 2.4 ([Scha, Corollary 5.8.3]). If $|D|=1$, then $G(D)=1$. If $|D|=p$ for some odd prime $p, D=\langle x\rangle$, then

$$
G(D)=\left\{\begin{array}{lll}
\left(\frac{q(x)}{p}\right) & \text { if } p \equiv 1 & (\bmod 4) \\
i\left(\frac{q(x)}{p}\right) & \text { if } p \equiv 3 & (\bmod 4)
\end{array} .\right.
$$

For $p=2$ one has 8 nonisometric anisotropic orthogonally indecomposable quadratic 2-groups: 
Lemma 2.5. Let $p=2$ and $(D, q)$ be a nontrivial anisotropic orthogonally indecomposable 2-group. Then one of the following three possibilities occurs:

(1) $D=\langle x, y\rangle \cong \mathbb{Z} / 2 \mathbb{Z} \times \mathbb{Z} / 2 \mathbb{Z}$ with $q(a x+b y)=\frac{1}{2}\left(a^{2}+a b+b^{2}\right)+\mathbb{Z}$. In this case $G(D, q)=-1$.

(2) $D=\langle x\rangle \cong \mathbb{Z} / 2 \mathbb{Z}$ with $q(x)=\frac{\epsilon}{4}+\mathbb{Z}$ where $\epsilon= \pm 1$. Here $G(D, q)=$ $\exp \left(\frac{2 \pi i}{8}\right)^{\epsilon}$.

(3) $D=\langle x\rangle \cong \mathbb{Z} / 4 \mathbb{Z}$ with $q(x)=\frac{\epsilon}{8}+\mathbb{Z}$ where $\epsilon= \pm 1, \pm 3$ and $G(D, q)=$ $\exp \left(\frac{2 \pi i}{8}\right)^{\epsilon}$.

Proof. By [Cas, Lemma 8.4.1], any regular quadratic 2-group $(D, q)$ is isometric to the orthogonal sum copies of

$$
\left(\mathbb{Z} /\left(2^{e-1} \mathbb{Z}\right)=\langle x\rangle, q(x)=\frac{a}{2^{e}}+\mathbb{Z}\right) \quad \text { with } a= \pm 1, \pm 3 \text { and } e \in \mathbb{N}
$$

and

$\left(\mathbb{Z} /\left(2^{e-1} \mathbb{Z}\right)^{2}=\langle x, y\rangle\right.$ with $q(a x+b y)=\frac{1}{2^{e}}\left\{\begin{array}{l}a b \\ \text { or } \\ a^{2}+a b+b^{2}\end{array}+\mathbb{Z}\right)$ and $e \in \mathbb{N}$.

One now easily finds the anisotropic quadratic spaces among these.

This explicit calculation of the Gauss sums has the following important consequence.

Lemma 2.6. Let $L$ be an even lattice of dimension $n$ with $n \equiv 2(\bmod 8)$. Then exactly one of the following holds:

(a) The number of primes $p \equiv 3(\bmod 4)$ such that $p$ divides $\operatorname{det}(L)$ to an odd power is odd.

(b) $2^{2}$ divides $\operatorname{det}(L)$ and the number of orthogonal components of type (2) or (3) in Lemma 2.5 in the discriminant group of any maximal even overlattice of $L$ is $\equiv 2(\bmod 4)$.

\section{The strongly perfect lattices of dimension 10}

In this section we prove the following theorem:

Theorem 3.1. There are exactly two similarity classes of strongly perfect lattices in dimension 10. They are represented by $K_{10}^{\prime}$ and its dual lattice $\left(K_{10}^{\prime}\right)^{*}$.

The lattices $K_{10}^{\prime}$ and $\left(K_{10}^{\prime}\right)^{*}$ are described in [Mar, Chap. VIII, Tableau $\left.5.9^{\prime}, 5.11\right] . \quad K_{10}^{\prime}$ has minimum 4 , determinant $2^{2} \cdot 3^{5}$ and kissing number $2 \cdot 135$. The rescaled dual lattice $\sqrt{6}\left(K_{10}^{\prime}\right)^{*}$ has minimum 6 , determinant $2^{8} \cdot 3^{5}$ and kissing number $2 \cdot 120$.

Lemma 3.2. The proper overlattices of $K_{10}^{\prime}$ or $\left(K_{10}^{\prime}\right)^{*}$ have a strictly smaller minimum. 
Proof. Let $\Lambda$ be such an overlattice of $K_{10}^{\prime}$ and $p:=\left[\Lambda: K_{10}^{\prime}\right]$ its index. Then by Rogers' bound ([CoS, Table 1.2])

$$
\frac{\min (\Lambda)^{10}}{p^{2} \operatorname{det}\left(K_{10}^{\prime}\right)} \leq \gamma_{10}^{10} \leq 3.5 \frac{\min \left(K_{10}^{\prime}\right)^{10}}{\operatorname{det}\left(K_{10}^{\prime}\right)} .
$$

If $\min (\Lambda)=\min \left(K_{10}^{\prime}\right)$, then $p=1$. Analogously one shows the lemma for $\left(K_{10}^{\prime}\right)^{*}$.

Because of this lemma, we only have to show Theorem 3.1 under the assumption that the strongly perfect lattice is generated by its minimal vectors.

Now let $\Lambda$ be a strongly perfect lattice. We assume that $\Lambda$ is rescaled such that $\min (\Lambda)=1$ and choose $X \subseteq \Lambda_{1}$ such that $X \cap(-X)=\emptyset$ and $X \cup(-X)=\Lambda_{1}$.

3.1. Some invariants. In this subsection we use the equalities in Section 2 , to determine $s:=|X|$ and $r:=\min \left(\Lambda^{*}\right)=\left(\gamma^{\prime}\right)^{2}(\Lambda)$.

By Lemma 2.2 one has $r \geq 4$. But in fact equality holds here, which means that $\Lambda$ is of minimal type in the sense of [Ven].

Lemma 3.3. $r=4$ and $n_{2}(\alpha)=0$ for all $\alpha \in\left(\Lambda^{*}\right)_{4}$.

Proof. Let $\alpha \in\left(\Lambda^{*}\right)_{r}$. By the bounds on $s$ and $r$ given in Subsection 2.1, one has

$$
C:=\sum_{i=2}^{r} \frac{i^{4}-i^{2}}{12} n_{i}(\alpha)=\frac{s r}{120}\left(\frac{r}{4}-1\right) \leq 3 .
$$

Since $\left(i^{4}-i^{2}\right)=i^{2}(i-1)(i+1)$ is divisible by 12 for all $i \in \mathbb{Z}, C$ is integral, $C=n_{2}(\alpha)+6 n_{3}(\alpha)+\ldots$ Therefore $n_{3}(\alpha)=n_{4}(\alpha)=\ldots=0$ and $n_{2}(\alpha) \leq 3$. Write $r=: p / q$ with $p, q \in \mathbb{N}$ and $\operatorname{gcd}(p, q)=1$. Assume that $p \neq 4 q$. Then $s=480 n_{2}(\alpha) q^{2} /(p(p-4 q))$. Since $\operatorname{gcd}(p, q)=1$ one has that $p$ divides $480 n_{2}$ and $q^{2}$ divides $s \leq 297$. One finds the following possibilities:

\begin{tabular}{|c|c|c|c|c|}
\hline$n_{2}$ & $s$ & $p$ & $q$ & $c$ \\
\hline 1 & 96 & 5 & 1 & $2 / 5$ \\
1 & 125 & 24 & 5 & $5 / 12$ \\
1 & 243 & 40 & 9 & $9 / 20$ \\
1 & 169 & 60 & 13 & $13 / 30$ \\
\hline 2 & 192 & 5 & 1 & $4 / 5$ \\
2 & 250 & 24 & 5 & $5 / 6$ \\
2 & 289 & 80 & 17 & $17 / 20$ \\
\hline 3 & 288 & 5 & 1 & $6 / 5$ \\
3 & 245 & 36 & 7 & $7 / 6$ \\
\hline
\end{tabular}


This table lists the possible values $n_{2}(\alpha), s, p, q$ that satisfy the conditions above. The last column gives the constant $c=\frac{s}{60}\left(\frac{r}{4}-1\right)$ of Lemma 2.1 .

Since $n_{i}(\alpha)=0$ for all $i \leq 3$, the conditions of Lemma 2.1 are satisfied and

$$
\sum_{x \in N_{2}(\alpha)} x=c \alpha
$$

where $c$ is the constant in the last column of the table above. If $n_{2}(\alpha)=1$, then $N_{2}(\alpha)=\{x\}$ and therefore

$$
c^{2}(\alpha, \alpha) \frac{p}{q}=1
$$

This is a contradiction in the four cases where $n_{2}(\alpha)=1$.

Since $N_{2}(\alpha)$ consists of minimal vectors (of square length 1 ) in a lattice, the scalar products $\left(x_{1}, x_{2}\right) \leq 1 / 2$ for all $x_{1}, x_{2} \in N_{2}(\alpha)$. Hence if $n_{2}(\alpha)=2$, then

$$
3 \geq\left(\sum_{x \in N_{2}(\alpha)} x, \sum_{x \in N_{2}(\alpha)} x\right)=c^{2}(\alpha, \alpha)=c^{2} p / q
$$

which leads to a contradiction in the cases where $n_{2}=2$.

In the remaining two cases $n_{2}=3$. Let $N_{2}(\alpha):=\{x, y, z\}$. Then $c(x, \alpha)=2 c=(x, x)+(x, y)+(x, z)$. Analogous equations for $y$ and $z$ show that

$$
2 c-1=(x, y)+(x, z)=(x, y)+(y, z)=(x, z)+(y, z)
$$

whence $(x, y)=(2 c-1) / 2>1 / 2$ which is a contradiction.

Therefore $n_{2}(\alpha)=0$ and $r=4$.

We now determine the possible values for $s$ :

Lemma 3.4. $s=5 s_{0}$ where $s_{0} \in\{24,32,27,25\}$.

Proof. From equation (D2), with $\alpha \in\left(\Lambda^{*}\right)_{4}$, one gets that $2 s / 5=n_{1}(\alpha) \in$ $\mathbb{Z}$. Therefore $s$ is divisible by 5 ,

$$
s=5 s_{0} .
$$

For any $\alpha \in \Lambda^{*}$ write $(\alpha, \alpha)=p / q$ with $p, q \in \mathbb{Z}, \operatorname{gcd}(p, q)=1$. Since $\frac{1}{12}((D 4)-(D 2)) \in \mathbb{Z}$ one gets that

$$
\frac{s_{0} p(p-4 q)}{2^{5} \cdot 3 q^{2}} \in \mathbb{Z}
$$

In particular $q^{2} \mid s_{0} \leq 45$, hence $q \in\{1,2,3,4,5,6\}$.

If $q$ is even, then $128=2^{7}$ divides $s_{0}$ which is a contradiction. Hence $q$ is odd.

If $q=5$ then $5^{2} \mid s_{0}$ and therefore $s_{0}=25$.

If $q=3$ then $3^{3} \mid s_{0}$ and therefore $s_{0}=27$. 
So assume that $q=1$, i.e. $(\alpha, \alpha) \in \mathbb{Z}$ for all $\alpha \in \Lambda^{*}$. If $4 \mid(\alpha, \alpha)$ for all $\alpha \in \Lambda^{*}$, then $1 / \sqrt{2} \Lambda^{*}=: \Gamma$ is an even lattice with $\min (\Gamma)=2$ and $\min \left(\Gamma^{*}\right)=2$. Hence $\Gamma_{2} \subseteq\left(\Gamma^{*}\right)_{2}$. But then for all $\alpha \in\left(\Lambda^{*}\right)_{4}$ one has $\frac{1}{2} \alpha \in \Lambda_{1}$ and $\left(\alpha, \frac{1}{2} \alpha\right)=2$ contradicts the statement $n_{2}(\alpha)=0$ of Lemma 3.3.

Therefore there is $\alpha \in \Lambda^{*}$ with $4 \nmid(\alpha, \alpha)$ and hence $8 \mid s_{0}, s_{0} \in\{8,16,24$, $32,40\}$.

If $2^{5}$ does not divide $s_{0}$ then $\Lambda^{*}$ is an even lattice. Assume also that $3 \nmid s_{0}$, hence $s_{0} \neq 24,32$. By $(D 22)$ for all $\alpha, \beta \in \Lambda^{*}$, one has

$$
\sum_{x \in X}(x, \alpha)^{2}(x, \beta)^{2}=(s / 120)\left(2(\alpha, \beta)^{2}+(\alpha, \alpha)(\beta, \beta)\right) .
$$

Since $3 \nmid s$, this implies that 3 divides $\left(2(\alpha, \beta)^{2}+(\alpha, \alpha)(\beta, \beta)\right)$ for all $\alpha, \beta \in$ $\Lambda^{*}$, and therefore

$$
\Gamma_{(3)}=\left\{v \in \Lambda^{*} \mid(v, v) \equiv 0 \quad(\bmod 3)\right\}=3 \Lambda \cap \Lambda^{*}
$$

is a sublattice of $\Lambda^{*}$ of index 3. Since $\sqrt{3}^{-1} \Gamma_{(3)}$ is an even lattice, the determinant $d:=\operatorname{det}\left(\Lambda^{*}\right)$ is divisible by $3^{10-2}=3^{8}$. But $\min (\Lambda)=1$ and $\operatorname{det}(\Lambda)=1 / d$ implies that

$$
\gamma(\Lambda)^{10}=d \leq\left(\gamma_{10}\right)^{10}<3719<3^{8}
$$

which is a contradiction.

The main result of this subsection is the next lemma, which is stated without proof in [Ven, Théorème 13.2].

Lemma 3.5. $s=120$ or $s=135$.

Proof. By Lemma 3.4, we have to exclude the cases $s=160$ and $s=125$. Assume first that $s=160$. Then by $(\star)$ the norms of all vectors in $\Lambda^{*}$ are integral and either 0 or 1 modulo 3 . We also have $\min \left(\Lambda^{*}\right)=4$ and $\min (\Lambda)=1$. Assume that there is $\alpha \in \Lambda^{*}$ with $(\alpha, \alpha)=6$. Then $n_{i}(\alpha)=0$ for all $i \geq 3$ and $n_{2}(\alpha)=4$. Let $N_{2}(\alpha)=\left\{x_{1}, x_{2}, x_{3}, x_{4}\right\}$. Then by Lemma $2.1 x:=x_{1}+x_{2}+x_{3}+x_{4}=4 / 3 \alpha$. But $16 / 9 \cdot 6=(x, x)>10$ contradicts the fact that $\left(x_{i}, x_{j}\right) \leq 1 / 2$ for all $1 \leq i \neq j \leq 4$. Therefore $\left(\Lambda^{*}\right)_{6}=\emptyset$. Let $\Gamma:=\sqrt{2} \Lambda^{*}$. Then $\Gamma$ is an even (and hence integral) lattice. Since 3 does not divide $s$ the equation $(\star \star)$ of the proof of Lemma 3.4 shows that $\Gamma_{(3)}$ is a sublattice of $\Gamma$ of index 3 , and $3^{8}$ divides $\operatorname{det}(\Gamma)=: d$. Since $\min \left(\Gamma_{(3)}\right) \geq 18$, one gets that

$$
d \geq \frac{18^{10}}{9 \gamma_{10}^{10}}>10^{8} .
$$

On the other hand $\min \left(\Gamma^{*}\right)=1 / 2$ and therefore

$$
d \leq\left(2 \gamma_{10}\right)^{10}<4 \cdot 10^{7}
$$


which is a contradiction.

Now assume that $s=125$. By equation $(\star)$ of the proof of Lemma 3.4 one has that $\Gamma:=\sqrt{5} / \sqrt{2} \Lambda^{*}$ is an even lattice. By $(\star \star)$, since $3 \nmid s$, the set $\Gamma_{(3)}$ is a sublattice of $\Gamma$ of index 3 .

Assume that there is a vector $\alpha \in\left(\Lambda^{*}\right)_{24 / 5}$. Then $n_{2}(\alpha)=7$ and $v:=$ $\sum_{x \in N_{2}(\alpha)} x=\frac{35}{12} \alpha$ by Lemma 2.1. Since the $x \in N_{2}(\alpha)$ are minimal vectors of $\Lambda$, one has $(v, v) \leq 7+7 \cdot 6 \cdot \frac{1}{2}=28$. But $(v, v)=\left(\frac{35}{12}\right)^{2} \frac{24}{5}>40$ which is a contradiction.

Therefore $\min \left(\Gamma_{(3)}\right) \geq 18$ and the determinant $d:=\operatorname{det}(\Gamma)$ satisfies

$$
10^{8}<\frac{18^{10}}{9 \cdot \gamma_{10}^{10}} \leq d \leq \frac{\gamma_{10}^{10} \cdot 5^{10}}{2^{10}}<4 \cdot 10^{7}
$$

which is a contradiction.

3.2. The case $s=120$. In this subsection we assume that $\Lambda$ is a strongly perfect lattice of minimum 1 and dimension 10 , such that $\left|\Lambda_{1}\right|=2 \cdot 120$. We also assume that $\Lambda$ is generated by its minimal vectors.

Let $\Gamma:=\Lambda^{*}$. Then equation $(\star)$ of the proof of Lemma 3.4 shows that $\Gamma$ is an even lattice of minimum 4.

We show the following theorem:

Theorem 3.6. Let $\Gamma$ be an even lattice of dimension 10 and of minimum $\geq 4$ such that the minimum of $\Gamma^{*}$ is $\geq 1$ and $\Gamma^{*}$ is generated by its minimal vectors. Then $\Gamma$ is isometric to either $K_{10}^{\prime}$ or $Q_{10}$.

The lattice $Q_{10}([\mathrm{CoS}])$, (denoted by $F_{5}$ in [Sou]) has minimum 4 , determinant $2^{2} 4^{4}$ and $2 \cdot 130$ vectors of norm 4 . The dual lattice of $Q_{10}$ is similar to $Q_{10}$ and not strongly perfect. Therefore all strongly perfect lattices with kissing number $2 \cdot 120$ are similar to $\left(K_{10}^{\prime}\right)^{*}$, which is strongly perfect.

Let $\Gamma$ be such a lattice as in the theorem and $d:=\operatorname{det}(\Gamma)$. Since $\min \left(\Gamma^{*}\right)=1$, one has $d \leq \gamma_{10}^{10}<3719$. Let $D:=\Gamma^{*} / \Gamma$. Then $q: D \rightarrow$ $\mathbb{Q} / \mathbb{Z} ; x+\Gamma \mapsto \frac{1}{2}(x, x)+\mathbb{Z}$ is a non degenerate quadratic form on the finite abelian group $D$. Since $\Gamma^{*}$ is generated by its minimal vectors, the group $D$ is generated by vectors $x$ with $q(x)=\frac{1}{2}+\mathbb{Z}$. Therefore one has:

Lemma 3.7. Let $p \neq 2$ be a prime and $D_{p}$ be the p-primary component of $D$. Then $D_{p}$ is not cyclic. If $\left|D_{p}\right|=p^{2}$ then $D_{p}$ is hyperbolic.

Proof. Let $|D|=l p^{\alpha}$ with $p \nmid l$. Then $D_{p}=l D$. Therefore $D_{p}$ is generated by the isotropic vectors $l x$ with $x \in X$. This implies that $D_{p}$ is not cyclic, and $D_{p}$ is hyperbolic, if $\alpha=2$.

We classify the lattices $\Gamma$ according to the 2 possibilities of Lemma 2.6:

Lemma 3.8. If $\Gamma$ satisfies case (b) of Lemma 2.6 then $\Gamma \cong Q_{10}$. 
Proof. Let $d=\operatorname{det}(\Gamma)$ be the determinant of $\Gamma$.

-We first claim that $2^{4}$ divides $d$, and $2^{6}$ divides $d$ if the maximal even overlattice of $\Gamma$ has determinant divisible by 8 . Let $D_{2}$ be the Sylow-2subgroup of the quadratic module $\left(\Gamma^{*} / \Gamma, q\right)$. Then $D_{2}$ is generated by elements $x$ with $q(x)=1 / 2+\mathbb{Z}$. The condition (b) of Lemma 2.6 implies that $D_{2}$ contains an element $y$ with $q(y) \in \frac{1}{8} \mathbb{Z}-\frac{1}{2} \mathbb{Z}$. Write $y=\sum_{i=1}^{t} x_{i}$ as sum of vectors $x_{i} \in D_{2}$ with $q\left(x_{i}\right)=1 / 2+\mathbb{Z}$. Then

$$
q(y)=\sum_{i=1}^{t} q\left(x_{i}\right)+\sum_{1 \leq i<j \leq t} b_{q}\left(x_{i}, x_{j}\right) .
$$

Therefore, there are vectors $x, x^{\prime} \in D_{2}$ with $b_{q}\left(x, x^{\prime}\right)= \pm 1 / 4+\mathbb{Z}$. Hence $\left\langle x, x^{\prime}\right\rangle \cong \mathbb{Z} / 4 \mathbb{Z} \times \mathbb{Z} / 4 \mathbb{Z}$ is a submodule of $D_{2}$ and therefore $2^{4}$ divides $d$.

If 8 divides the determinant of a maximal even overlattice of $\Gamma$, then $D_{2}$ contains an element $y$ with $q(y)=\epsilon / 8+\mathbb{Z}$, with $\epsilon= \pm 1, \pm 3$. One concludes that there is a pair of elements $x, x^{\prime} \in D_{2}, q(x)=q\left(x^{\prime}\right)=1 / 2+\mathbb{Z}$ and $b_{q}\left(x, x^{\prime}\right)=1 / 8+\mathbb{Z}$. The order of $\left\langle x, x^{\prime}\right\rangle$ is at least $2^{6}$.

Now $d / 2^{4}<3719 / 2^{4}<233$ and hence all the primes $p$ that divide $d$ are $\leq 13$. Moreover if a prime $p>3$ divides $d$ then $p^{2}$ is the largest $p$-power dividing $d$ and $D_{p}$ is hyperbolic. If $3^{3}$ divides $d$ then also $3^{4}$ divides $d$ and $d=2^{4} \cdot 3^{4}$.

- Let $L$ be a maximal even overlattice of $\Gamma$. Then $\operatorname{det}(L)=2^{2}$ and $L$ is in the genus of $D_{10}, \operatorname{det}(L)=4 \cdot 2$ and $L$ is in the genus of $E_{7} \perp D_{3}$ or $\operatorname{det}(L)=2^{2} \cdot 3^{2}$ and $L$ is in the genus of $A_{5}^{2}$. Calculating the respective genera with [MAG] one sees that $\min \left(L^{*}\right) \geq 1$ implies that $L \cong D_{10}$.

-We claim that no prime $p>2$ divides $d$. Otherwise $\Gamma^{*}$ contains a lattice $M^{*}=\left\langle D_{10}^{*}, v\right\rangle$ with $M^{*} / D_{10}^{*}=p$. In the coordinates with respect to an orthonormal basis of the sublattice $\mathbb{Z}^{10}$ of $D_{10}$ write $v=1 / p\left(a_{1}, \ldots, a_{10}\right)$ with $a_{i} \in \mathbb{Z}$ and $\left|a_{i}\right| \leq(p-1) / 2$. Let $n_{i}:=\mid\left\{1 \leq j \leq 10|| a_{j} \mid=i\right\}$ for $i=0, \ldots, \frac{p-1}{2}$. Then

$$
\sum_{i=1}^{10} a_{i}^{2}=\sum_{i=1}(p-1) / 2 i^{2} n_{i} \geq p^{2} .
$$

Multiplying by the integers $2, \ldots, p-1$ and reducing the coefficient $a_{i} / p$ modulo $\mathbb{Z}$, one finds in total $(p-1) / 2$ inequalities $(1)$, where the $n_{i}$ are permuted cyclicly. Summing them up, one finds

$$
\left(\sum_{i=1}^{(p-1) / 2} i^{2}\right)\left(n_{1}+\ldots+n_{(p-1) / 2}\right) \geq \frac{p-1}{2} p^{2}
$$

If $p \geq 7$ this implies that $n_{1}+\ldots+n_{(p-1) / 2} \geq 11$ which is a contradiction. For $p=3,5$ one checks by hand, that there are no such lattices, using the 
fact that

$$
\left\|v-\left( \pm \frac{1}{2}\right)^{10}\right\|^{2}=\sum_{i=0}^{10}\left(2 a_{i}-p\right)^{2} \geq 4 \cdot p^{2}
$$

- One finds 336 overlattices of $D_{10}^{*}$ of index 2 with minimum 1, which fall into 2 isometry classes, represented by, say, $L$ and $L^{\prime}$. The overlattices of $L$ and $L^{\prime}$ with minimum 1 and index 2 fall into 3 isometry classes. These three lattices have up to isometry a unique overlattice, say $M^{*}$, of index 2 , minimum 1. The lattice $M$ is isometric to $Q_{10}$. As in the proof of Lemma 3.2 one sees that $M^{*}$ has no proper overlattice of minimum 1 .

Lemma 3.9. If $\Gamma$ satisfies case (b) of Lemma 2.6 then $\Gamma \cong K_{10}^{\prime}$.

Proof. Let $\Gamma$ be such a lattice.

- We first show that $2^{2}$ divides $d$ : Let $\Gamma^{\prime}:=\langle\Gamma, x\rangle$ for any $x \in X$. Then $\Gamma^{\prime}$ is an integral lattice with $2 \mid\left[\Gamma^{\prime}: \Gamma\right]$. Therefore $2^{2}\left|\left[\Gamma^{*} /\left(\Gamma^{\prime}\right)^{*}\right]\left[\Gamma^{\prime}: \Gamma\right]\right| d$.

Since $d<3719$ and $p^{3}$ divides $d$ for some prime $p \equiv 3(\bmod 4)$, one has that either $7^{3} \mid d$ or $3^{3} \mid d$. In the first case $d=7^{3} \cdot 2^{2}$ or $d=7^{3} \cdot 2^{3}$. In the second case $d=2^{2} \cdot 3^{3} d_{0}$ with $d_{0}<34$ and all odd primes divide $d_{0}$ to an even power. Therefore one finds that

- $d=\operatorname{det}(\Gamma)$ is one of the following:

$$
2^{2} 7^{3}, 2^{3} 7^{3}, 2^{2} 3^{5}, 2^{3} 3^{5}, 2^{a} 3^{3}(a=2, \ldots, 7) \text {, or } 2^{2} 3^{3} 5^{2} .
$$

- The maximal even overlattices of $\Gamma$ are all isometric to $D_{4} \perp E_{6}$ : Let $L$ be a maximal even overlattice of $\Gamma$. Then $\operatorname{det}(L)$ is one of $7,2^{2} \cdot 7,2^{3} \cdot 7,3,2^{2} \cdot 3$, or $2^{3} \cdot 3$ and for each determinant there is only one genus of maximal even lattices. If $\operatorname{det}(L)=2^{3} \cdot 7$, then the Sylow 2-subgroup of $\Gamma^{*} / \Gamma$ is isometric to the one of $L^{*} / L$. But the latter does not contain an isotropic vector, which contradicts the fact that $2 x$ is isotropic for all $x \in\left(\Gamma^{*}\right)_{1}$. Therefore $\operatorname{det}(L) \neq 2^{3} \cdot 7$. For the other determinants one can list all the lattices in the genus (e.g. with $[\mathrm{MAG}]$ ). The property that $\min \left(L^{*}\right) \geq 1$ excludes all possibilities except for $L \cong D_{4} \perp E_{6}$.

-There is a unique orbit of overlattices of index 3 of $\left(D_{4} \perp E_{6}\right)^{*}$ under the automorphism group that consists of lattices of minimum $\geq 1$. Let $M^{*}$ be such a lattice. All the overlattices of $M^{*}$ one index 2 or 5 contain vectors of length $<1$. Therefore $\Gamma^{*}$ is an overlattice of $M^{*}$ of index 3 , and there are only 48 such lattices of minimum $\geq 1$. All these lattices are isometric to $\left(K_{10}^{\prime}\right)^{*}$.

3.3. The case $s=135$. In this section we prove the following

Theorem 3.10. Let $\Lambda$ be a strongly perfect lattice of dimension 10 with $2 \cdot 135$ minimal vectors. Then $\Lambda$ is similar to $\left(K_{10}^{\prime}\right)^{*}$. 
Let $\Lambda$ be a strongly perfect lattice of minimum 1 and dimension 10 , such that $\left|\Lambda_{1}\right|=2 \cdot 135$. We assume again that $\Lambda$ is generated by its minimal vectors. Equation $(\star)$ of the proof of Lemma 3.4 shows that

$$
\Gamma:=\frac{\sqrt{3}}{\sqrt{2}} \Lambda^{*}
$$

is an even lattice of minimum 6 . Let $d:=\operatorname{det}(\Gamma)$. Since $\min \left(\Gamma^{*}\right)=2 / 3$, one has $d \leq\left(\frac{3}{2}\right)^{10} \gamma_{10}^{10}<214439$. Let $D:=\Gamma^{*} / \Gamma$.

Lemma 3.11. $\Gamma_{(4)} \subseteq \Gamma \cap 2 \Gamma^{*}$ is a sublattice of $\Gamma$ of index 2 or 4.

Proof. Since $(D 13)$ and $(D 11)$ hold for all $\alpha_{2} \in \mathbb{R}^{10}$, one finds that for all $\alpha \in \mathbb{R}^{10}$

$$
\sum_{x \in X}(x, \alpha)^{3} x=\frac{3}{2}(\alpha, \alpha) \alpha \text { and } \sum_{x \in X}(x, \alpha) x=9 \alpha .
$$

In particular if $\alpha \in \Gamma$, then $(x, \alpha)^{3}-(x, \alpha)$ is divisible by 6 and hence $\left(\frac{3}{2}(\alpha, \alpha)-9\right) \alpha \in 6 \Gamma^{*}$. If 4 divides $(\alpha, \alpha)$ then this shows that $\alpha \in 2 \Gamma^{*}$, and hence $\Gamma_{(4)} \subseteq \Gamma \cap 2 \Gamma^{*}$. In particular $(\alpha, \beta)$ is even for all $\alpha, \beta \in \Gamma_{(4)}$ and $\Gamma_{(4)}$ is a lattice.

Let $\alpha_{1}, \alpha_{2} \in \Gamma-\Gamma_{(4)}$. Then $\alpha_{1}-\alpha_{2} \in \Gamma_{(4)}$ if and only if $\left(\alpha_{1}, \alpha_{2}\right)$ is even. Since $\min \left(\Gamma^{*}\right)^{2} \frac{s}{n(n+2)}=\frac{4 \cdot 135}{120}=\frac{9}{2}$ the equality $(D 1111)$ shows that for all $\alpha_{1}, \ldots, \alpha_{4} \in \Gamma$

$$
\left(\alpha_{1}, \alpha_{2}\right)\left(\alpha_{3}, \alpha_{4}\right)+\left(\alpha_{1}, \alpha_{3}\right)\left(\alpha_{2}, \alpha_{4}\right)+\left(\alpha_{1}, \alpha_{4}\right)\left(\alpha_{2}, \alpha_{3}\right) \in 2 \mathbb{Z} .
$$

Hence $\left|\Gamma / \Gamma_{(4)}\right| \leq 4$. Since $\Gamma \neq \Gamma_{(4)}$ and the index is a power of 2 , one has $\left|\Gamma / \Gamma_{(4)}\right|=2$ or 4 .

Corollary 3.12. $2^{8} \mid d=\operatorname{det}(\Gamma)$.

Proof. Let $\Gamma_{(4)}$ be as in Lemma 3.11 and $\alpha \in \Gamma-\Gamma_{(4)}$. Since $\Gamma_{(4)} \subseteq 2 \Gamma^{*}$, all the scalar products in the lattice $\Gamma^{\prime}:=\left\langle\Gamma_{(4)}, \alpha\right\rangle$ are divisible by 2 . Therefore $2^{10}$ divides $\operatorname{det}\left(\Gamma^{\prime}\right)$. Since $\left[\Gamma: \Gamma^{\prime}\right] \leq 2,2^{8}$ divides $d$.

Since the norms of the minimal vectors in $\Gamma^{*}$ are $2 / 3$, it is clear that 3 divides $d$. Moreover, if $2^{9}$ divides $d$, then also $2^{10}$ divides $d$. If we are in case (b) of Lemma 2.6, then the argument of Lemma 3.8 shows that also $2^{10}$ divides $d$. Hence we have the following possibilities for $d$ :

Lemma 3.13. If $\Gamma$ satisfies case (a) of Lemma 2.6, then $\operatorname{det}(\Gamma)$ is one of $2^{8} \cdot 3 \cdot 13^{2}, 2^{8} \cdot 3 \cdot 11^{2}, 2^{8} \cdot 3 \cdot 7^{2}, 2^{10} \cdot 3 \cdot 7^{2}, 2^{8} \cdot 3 \cdot 5^{3}, 2^{8} \cdot 3 \cdot 5^{2}, 2^{10} \cdot 3 \cdot 5^{2}$, $2^{11} \cdot 3 \cdot 5^{2}, 2^{8} \cdot 3^{3} \cdot 5^{2}, 2^{8} \cdot 3^{5}, 2^{8+a} \cdot 3^{3}, 2^{8+b} \cdot 3$, where $0 \leq a \leq 4$ and $0 \leq b \leq 8$.

If $\Gamma$ satisfies case (b) of Lemma 2.6, then $\operatorname{det}(\Gamma)$ is one of

$$
2^{10} \cdot 3^{4}, 2^{11} \cdot 3^{4}, 2^{10+a} \cdot 3^{2}, \text { where } 0 \leq a \leq 4 .
$$


If one could prove the existence of a norm 10 vector in $\Gamma$, then this would exclude most of the possible determinants. But unfortunately, we did not succeed in proving this directly.

Remark 3.14. If $\Gamma$ contains a vector of square length 10 , then $3^{4}$ divides $\operatorname{det}(\Gamma)$.

Proof. Let $\alpha \in \Gamma$ with $(\alpha, \alpha)=10$. Since $10 \frac{2}{3}<9$, the scalar products $(\alpha, x)$ with $x \in X$ are $0, \pm 1, \pm 2$. By Lemma $2.1\left|N_{2}(\alpha)\right|=5$ and $\sum_{x \in N_{2}(\alpha)} x=\alpha$. Calculating the norm, one finds that

$$
10=(\alpha, \alpha)=\left(\sum_{x \in N_{2}(\alpha)} x, \sum_{x \in N_{2}(\alpha)} x\right) \leq 5 \cdot \frac{2}{3}+5 \cdot 4 \cdot \frac{1}{3}=10
$$

since the vectors in $N_{2}(\alpha)$ are minimal vectors in a lattice. Therefore $(x, y)=\frac{1}{3}+\delta_{x y} \frac{1}{3}$ for all $x, y \in N_{2}(\alpha)$ and $N_{2}(\alpha)$ generates a lattice isomorphic to $\sqrt{3}^{-1} A_{5}$ of determinant $2 \cdot 3^{-4}$. In particular one has an epimorphism $\mathbb{Z}_{3} \otimes_{\mathbb{Z}}\left\langle\Gamma, N_{2}(\alpha)\right\rangle \rightarrow\left(\mathbb{Z}_{3} \otimes_{\mathbb{Z}} \sqrt{3}^{-1} A_{5}\right) /\left(\mathbb{Z}_{3} \otimes_{\mathbb{Z}} A_{5}\right)^{*}$ which contains $\mathbb{Z}_{3} \otimes \Gamma$ in its kernel. Therefore the order of the discriminant group $\Gamma^{*} / \Gamma$ is divisible by $3^{4}$.

Lemma 3.15. There is an even overlattice $\Gamma^{\prime}:=\left\langle 3 x_{1}, 3 x_{2}, \Gamma\right\rangle$ isometric to an even orthogonally decomposable lattice $\Gamma^{\prime} \cong A_{2} \perp L$ with $\Gamma \subseteq \Gamma^{\prime} \subseteq$ $\Gamma+3 \Gamma^{*}$.

Proof. Assume first that there are no vectors of norm 8 in $\Gamma$. Then the minimum of the lattice $\Gamma_{(4)}$ of Lemma 3.11 is 12 . Since the index of $\Gamma_{(4)}$ in $\Gamma$ is at most 4 , one finds $12 /(4 d)^{1 / 10} \leq \gamma_{10}$, hence $d \geq 12^{10} /\left(4 \gamma_{10}\right)^{10}>10^{6}$. This contradicts the fact that $\operatorname{det}(\Gamma) \leq\left(\frac{3}{2}\right)^{10} \gamma_{10}^{10}<214439$. Therefore there is a vector $\alpha \in \Gamma$ with $(\alpha, \alpha)=8$.

One calculates that $n_{2}(\alpha)=2$ and $2\left(x_{1}+x_{2}\right)=\alpha$ where $N_{2}(\alpha)=$ $\left\{x_{1}, x_{2}\right\}$. Since $\left(x_{i}, x_{i}\right)=2 / 3$ and the $x_{i}$ are minimal vectors in $\Gamma^{*}$, one gets $\left(x_{i}, x_{j}\right)=1 / 3$. Let $\Gamma^{\prime}:=\left\langle 3 x_{1}, 3 x_{2}, \Gamma\right\rangle$. Then $\Gamma^{\prime}$ is an even lattice and $L^{\prime}:=\left\langle 3 x_{1}-\alpha, 3 x_{2}-\alpha\right\rangle \leq \Gamma^{\prime}$ is a sublattice of $\Gamma^{\prime}$ isometric to $A_{2}$. Moreover $\left(x_{i}, 3 x_{j}-\alpha\right)=\delta_{i j}-1$. So $\left\langle x_{1}, x_{2}\right\rangle \leq\left(\Gamma^{\prime}\right)^{*}$ generate the dual lattice of $L^{\prime}$ and hence $\Gamma^{\prime} \cong A_{2} \perp L$ for some 8-dimensional even lattice $L$.

Lemma 3.16. The maximal even overlattice of the form $A_{2} \perp L$ of $\Gamma$ is $A_{2} \perp L_{0}$, with $L_{0}$ isometric to one of

$$
E_{8}, D_{4} \perp A_{4}, D_{4} \perp A_{2} \perp A_{2} \text {, or } D_{6} \perp A_{2} .
$$

Proof. Using the fact that the rank of the Sylow-2-subgroup of the discriminant group of an even lattice is congruent to the dimension modulo 2 , one finds with Lemma 3.13 that the possible determinants of $L_{0}$ are $1,2^{2}, 5,2^{2} \cdot 5,2 \cdot 4,3^{2}, 2^{2} \cdot 3^{2}, 2 \cdot 4 \cdot 3^{2}, 2^{2} \cdot 3$, and $2 \cdot 4 \cdot 3$. If $\operatorname{det}\left(L_{0}\right)=2^{2}$, then 
the discriminant group is isometric to the anisotropic group of Lemma 2.5 (1) and $\gamma\left(L_{0}\right)=-1 \neq(-1)^{8}$ contradicting Lemma 2.3. Similarly, there is no maximal even 8-dimensional lattice of determinant $3^{2}$. For all the other determinants there is a unique genus of maximal even lattices $L_{0}$. If $\operatorname{det}\left(L_{0}\right)=1$, then clearly $L_{0} \cong E_{8}$. There is a unique even 8-dimensional lattice of determinant 5 , but its dual has minimum $2 / 5<2 / 3$, therefore $\operatorname{det}\left(L_{0}\right) \neq 5$. The lattices $L_{0}$ of determinant $2^{2} .5$ ly in the genus of $D_{4} \perp A_{4}$. This genus consists of 2 lattices, but only for $D_{4} \perp A_{4}$, the dual lattice has minimum $\geq 2 / 3$. There are 2 lattices $L_{0}$ of determinant $4 \cdot 2$, namely $A_{1} \oplus D_{7}$ and $\sqrt{2} A_{1} \oplus E_{7}$, for both of which the dual lattice has minimum $<2 / 3$. If $\operatorname{det}\left(L_{0}\right)=2^{2} \cdot 3^{2}$, then $L_{0}$ lies in the genus of $A_{2} \perp A_{2} \perp D_{4}$. This genus consists of 3 lattices, but only for $A_{2} \perp A_{2} \perp D_{4}$, the dual lattice has minimum $\geq 2 / 3$. There is a unique genus of maximal even lattices $L_{0}$ with $\operatorname{det}\left(L_{0}\right)=2 \cdot 4 \cdot 3^{2}$, namely the one of $A_{3} \perp A_{2} \perp A_{2} \perp A_{1}$. It consists of 8 lattices, of which all the dual lattices contain vectors of norm $<2 / 3$. In the last 2 cases $\operatorname{det}\left(L_{0}\right)=2^{2} \cdot 3$ or $2 \cdot 4 \cdot 3, \Gamma$ satisfies the alternative (b) of Lemma 2.6. There are in total 5 lattices in the two genera, only one of which, $D_{6} \perp A_{2}$, has a dual lattice of minimum $\geq 2 / 3$.

The most complicated case is $L_{0} \cong E_{8}$. Since there is an even overlattice $A_{2} \perp L$ of $\Gamma$ generated by 2 vectors and the 2 -rank of $\Gamma^{*} / \Gamma$ is at least 8 , the 2-rank of the discriminant group of $L$ is at least 4 . By computer calculation one checks:

Lemma 3.17. The sublattices $L$ of $E_{8}$ such that $\min \left(L^{*}\right) \geq 2 / 3$ and the 2-rank of $L^{*} / L$ is at least 4 are contained in $D_{4} \perp D_{4}$.

Lemma 3.18. No prime $p \geq 7$ divides $\operatorname{det}(\Gamma)$.

Proof. Assume that a prime $p \geq 7$ divides $\operatorname{det}(\Gamma)$. Then $\operatorname{det}(\Gamma)$ is one of $2^{8} \cdot 3 \cdot 13^{2}, 2^{8} \cdot 3 \cdot 11^{2}, 2^{8} \cdot 3 \cdot 7^{2}$, or $2^{10} \cdot 3 \cdot 7^{2}$. In particular the maximal even overlattice of Lemma 3.16 of $\Gamma$ is $A_{2} \perp E_{8}$. By Lemma $3.17 \Gamma^{*}$ is an overlattice of $L^{*}:=A_{2}^{*} \perp D_{4}^{*} \perp D_{4}^{*}$. We choose coordinates for this lattice, such that $v:=\left(a_{1}, \ldots, a_{10}\right)$ has norm $(v, v):=\frac{2}{3}\left(a_{1}^{2}+a_{1} a_{2}+a_{2}^{2}\right)+\sum_{i=3}^{10} a_{i}^{2}$. Note that the vectors with integral coordinates ly in $L^{*}$. Assume that $p=7,11$, or 13 divides $\operatorname{det}(\Gamma)$. Then $\Gamma^{*}$ contains a lattice $\left\langle L^{*}, \frac{1}{p} v\right\rangle$, where the coordinates of $v$ are $\left(a_{1}, \ldots, a_{10}\right) \in \mathbb{Z}^{10}$ with $-\frac{p-1}{2} \leq a_{i} \leq \frac{p-1}{2}$. Since the Sylow- $p$-subgroup of $\Gamma^{*} / \Gamma$ is a hyperbolic plane, we may also assume that $\frac{1}{p} v$ is isotropic, i.e. $p^{2} \mid(v, v)$. Since $\min \left(\Gamma^{*}\right)=2 / 3$ we have that $(v, v) \geq p^{2}$. Now $\frac{2}{3}\left(a_{1}^{2}+a_{1} a_{2}+a_{2}^{2}\right) \leq a_{1}^{2}+a_{2}^{2}$, because the difference is $\frac{1}{3}\left(a_{1}^{2}-2 a_{1} a_{2}+a_{2}^{2}\right)=\frac{1}{3}\left(a_{1}-a_{2}\right)^{2} \geq 0$. Therefore $(v, v) \leq \sum_{i=1}^{10} a_{i}^{2}=: q^{+}(v)$. Working with this bigger quadratic form $q^{+}$we can argue as in the proof of Lemma 3.8: Let $n_{i}:=\mid\left\{1 \leq j \leq 10|| a_{j} \mid=i\right\}$ for $i=0, \ldots \frac{p-1}{2}$. Then 
$\left(\sum_{i=1}^{(p-1) / 2} i^{2}\right)\left(n_{1}+\ldots+n_{(p-1) / 2}\right) \geq \frac{p-1}{2} p^{2}$ implies that $n_{1}+\ldots+n_{(p-1) / 2} \geq 11$ which is a contradiction.

Proof of Theorem 3.10. It remains to consider the sublattices of $A_{2} \perp L_{0}$ for the lattices $L_{0}$ in Lemma 3.16 that have one of the determinants not divisible by 7,11 , or 13 that are listed in Lemma 3.13 .

-We first consider the case that $L_{0} \cong D_{6} \perp A_{2}$. Then we are in case (b) of Lemma 2.6 and hence $2^{10}$ divides the determinant of $\Gamma$. Since $2^{4} \chi \operatorname{det}\left(L_{0}\right)$, $\Gamma$ is contained in a lattice $A_{2} \perp L$ with $\left[L_{0}: L\right]=2$. One finds 3 isometry classes of such lattices $L$, such that $\min \left(L^{*}\right) \geq 2 / 3$. Calculating sublattices of index 2 and testing isometry, one finds 22 sublattice of index 4 of $A_{2} \perp L_{0}, 32$ of index 8 and 8 of index 16, such that the minimum of the dual lattices is $\geq 2 / 3$. The latter 8 lattices have determinant $3^{2} \cdot 2^{10}$ and minimum $\leq 4$. Therefore $\Gamma$ is of index 2 or 3 of one of those 8 lattices, but one finds no such sublattices such that the minimum of the dual lattice is $\geq 2 / 3$. Therefore $L_{0} \not A_{2} \perp D_{6}$.

In all the other cases we are in case (a) of Lemma 2.6.

- Now assume that $L_{0} \cong D_{4} \perp A_{2} \perp A_{2}$. One finds 2 sublattices $L$ of index 2 of $L_{0}$ where the dual lattice has minimum $\geq 2 / 3 . A_{2} \perp L$ has 13 sublattices $L^{\prime}$ of index 2 and 4 sublattices $L^{\prime}$ of index 4 with $\min \left(\left(L^{\prime}\right)^{*}\right) \geq$ $2 / 3$. The latter 4 lattices have determinant $2^{8} 3^{3}$ but the Sylow-2-subgroup of the discriminant group has only rank 6 . So $\Gamma$ is contained in one of these lattices of index divisible by 2 . One finds no sublattices $M$ of these 4 lattices of index 2 with $\min \left(M^{*}\right) \geq 2 / 3$. So also this case is impossible.

- If $L_{0} \cong D_{4} \perp A_{4}$ then $\operatorname{det}(\Gamma)=2^{8} \cdot 3 \cdot 5^{3}$ and $\Gamma$ is a sublattice $M$ of index 5 of one of the 9 sublattices $L$ of $A_{2} \perp D_{4} \perp A_{4}$ with $\min \left(L^{*}\right)=2 / 3$ and $L^{*} / L \cong \mathbb{F}_{2}^{8} \times \mathbb{F}_{3} \times \mathbb{F}_{5}$. With the computer one finds no such sublattices $M$ such that $M^{*} / M$ has an elementary abelian Sylow 5-subgroup and $\min \left(M^{*}\right)=2 / 3$. Therefore $5^{3}$ does not divide $\operatorname{det}(\Gamma)$.

- The hardest case is that $L_{0} \cong E_{8}$. By Lemma $3.17, \Gamma$ is contained in $A_{2} \perp D_{4} \perp D_{4}$ of index divisible by 4 . One finds 36 isometry classes of sublattices $L$ of index 4 in $A_{2} \perp D_{4} \perp D_{4}$ such that $\min \left(L^{*}\right) \geq 2 / 3,5$ of which satisfy $L^{*} / L \cong \mathbb{F}_{2}^{8}$. If $2^{8}$ is the highest 2 -power that $\operatorname{divides} \operatorname{det}(\Gamma)$, i.e. $\operatorname{det}(\Gamma)$ is one of $2^{8} \cdot 3 \cdot 5^{2}, 2^{8} \cdot 3^{3} \cdot 5^{2}, 2^{8} \cdot 3^{5}, 2^{8} \cdot 3^{3}, 2^{8} \cdot 3$ then $\Gamma$ is contained in one of these five lattices $M$ of index $5,3 \cdot 5,3^{2}, 3$, or 1 . Since all the lattices $M$ have vectors of length 4 , the latter case is impossible. One finds no sublattices $N$ of index 5 of the lattices $M$ such that $N^{*}$ has minimum $\geq 2 / 3$. So the first two cases are also impossible. The lattices $M$ have 2 sublattices $N$ of index 3 , such that the minimum of the dual lattice is $\geq 2 / 3$. These two lattices still contain vectors of length 4 . Therefore $\Gamma$ is a sublattice of index 3 of one of these two lattices. Up to isometry there is a unique such lattice $\Gamma$ such that $\min \left(\Gamma^{*}\right) \geq 2 / 3$. This lattice $\Gamma$ is similar to $\left(K_{10}^{\prime}\right)^{*}$. 
If $2^{10}$ divides $\operatorname{det}(\Gamma)$, then $\Gamma$ is contained in one of the (up to isometry) 60 sublattices $M$ of index 2 in the 36 lattices $L$ above that have $\min \left(M^{*}\right) \geq 2 / 3$. Only for 8 of the 60 lattices $M$ the Sylow 2-subgroup of the discriminant group is of rank $\geq 8$.

If $2^{10}$ is an exact divisor of $\operatorname{det}(\bar{\Gamma})$, i.e. $\operatorname{det}(\Gamma)$ is one of $2^{10} \cdot 3 \cdot 5^{2}, 2^{10} \cdot 3^{3}$, $2^{10} \cdot 3$, then $\Gamma$ is a sublattice of one of these 8 lattices $N$ of index 5,3 , or 1 . Since all the lattices $N$ contain vectors of length 4 , the last case is impossible. One finds no sublattices $O$ of index 3 or 5 of $N$ such that $\min \left(O^{*}\right) \geq 2 / 3$. Therefore this case is impossible.

The 60 lattices $M$ above contain up to isometry 14 sublattices $O$ of index 2 such that $\min \left(O^{*}\right) \geq 2 / 3$. All these lattices have vectors of length 4 , and one finds no sublattices of indes 2 or 3 of these lattices such that the minimum of the dual lattice is $\geq 2 / 3$. So we finally proved Theorem 3.10 .

\section{References}

[BaV] C. BACHOC, B. VENKOV, Modular forms, lattices and spherical designs. In [EM].

[Cas] J.W.S. CASSELS, Rational quadratic forms. Academic Press (1978).

[CoS] J.H. Conway, N.J A. SlOANE, Sphere Packings, Lattices and Groups. 3rd edition, Springer-Verlag (1998).

[CoS] J.H. Conway, N.J.A. SlOANe, On Lattices Equivalent to Their Duals. J. Number Theory 48 (1994), 373-382.

[EM] Réseaux euclidiens, designs sphériques et groupes. Edited by J. Martinet. Enseignement des Mathématiques, monographie 37, to appear.

[MAG] The Magma Computational Algebra System for Algebra, Number Theory and Geometry. available via the magma home page http://www.maths .usyd.edu . au:8000/u/magma/.

[Mar] J. MARTINET, Les Réseaux parfaits des espaces Euclidiens. Masson (1996).

[Mar1] J. MARTINET, Sur certains designs sphériques liés à des réseaux entiers. In [EM].

[MiH] J. Milnor, D. Husemoller, Symmetric bilinear forms. Springer-Verlag (1973).

[Scha] W. ScharlaU, Quadratic and Hermitian Forms. Springer Grundlehren 270 (1985).

[Sou] B. SouvigniER, Irreducible finite integral matrix groups of degree 8 and 10. Math. Comp. 61207 (1994), 335-350.

[Ven] B. VenKov, Réseaux et designs sphériques. Notes taken by J. Martinet of lectures by B. Venkov at Bordeaux (1996/1997). In [EM].

Gabriele NEBE

Abteilung Reine Mathematik

Universität Ulm

$89069 \mathrm{Ulm}$

Germany

E-mail : nebe@mathematik.uni-ulm.de

Boris Venkov

St. Petersburg Branch of the Steklov Mathematical Institute

Fontanaka 27

191011 St. Petersburg

Russia

E-mail : bvenkov@pdmi.ras.ru 